\title{
Efecto de dietas con alta concentración de Saccharomyces cerevisiae sobre la proliferación de hemocitos en camarones Cryphiops caementarius machos
}

\section{Effect of diets with high concentration of Saccharomyces cerevisiae on the proliferation of hemocytes in male shrimp Cryphiops caementarius}

\author{
Elizabeth Cabrera P., Zanny Marcelo T. ${ }^{1}$, Walter Reyes A., ${ }^{1,3}$, Carlos Azañero D. ${ }^{2}$
}

\section{Resumen}

\begin{abstract}
El objetivo del estudio fue evaluar el efecto de dietas con alta concentración de Saccharomyces cerevisiae sobre la proliferación de hemocitos en camarones Cryphiops caementarius machos. Los camarones $(5.3 \pm 1.2 \mathrm{~cm}$ de longitud total y $7.4 \pm 2.9 \mathrm{~g}$ de peso total) se colectaron del río Pativilca (Perú). Cada camarón se mantuvo en un recipiente instalado dentro del acuario (seis camarones por acuario). Se empleó una dieta control ( $3 \%$ de levadura) y tres dietas experimentales $(6,9$ y $12 \%$ de levadura) con dos repeticiones por tratamiento durante 28 días de cultivo. El número total de hemocitos fue mayor $(\mathrm{p}<0.05)$ con $6 \%$ de levadura $\left(134.75 \times 10^{5} \mathrm{cél} / \mathrm{ml}\right)$, así mismo el número de granulocitos $\left(31.44 \times 10^{5} \mathrm{cél} / \mathrm{ml}\right)$ y semigranulocitos $\left(102.44 \times 10^{5} \mathrm{cél} / \mathrm{ml}\right)$. El número de hialinocitos disminuyó en todos los tratamientos y se mantuvo entre 0.31 y 1.56 x $10^{5}$ cél $/ \mathrm{ml}$. El número de hemocitos atípicos se mantuvieron bajos en todos los tratamientos $\mathrm{y}$ sin diferencias con el basal $\left(0.31 \times 10^{5}\right.$ cél $\left./ \mathrm{ml}\right)$. La dieta con $6 \%$ de levadura incrementó $(\mathrm{p}<0.05)$ el número total de hemocitos y los hemocitos granulocitos y semigranulocitos en los camarones machos C. caementarius. En cambio, las dietas con 9 y $12 \%$ de levadura afectaron $(\mathrm{p}<0.05)$ la proliferación de hemocitos totales y diferenciales.
\end{abstract}

Palabras clave: Saccharomyces; Cryphiops; hemocitos; camarón; hematopoyesis

\footnotetext{
${ }^{1}$ Laboratorio de Acuicultura Ornamental, Departamento Académico de Biología, Microbiología y Biotecnología, Universidad Nacional del Santa, Nuevo Chimbote, Ancash, Perú

${ }^{2}$ Laboratorio de Microbiología y Bioquímica, Departamento Académico de Biología, Microbiología y Biotecnología, Universidad Nacional del Santa, Nuevo Chimbote, Ancash, Perú

${ }^{3}$ E-mail:wreyes_avalos@hotmail.com
} 
The aim of this study was to evaluate the effect of diets with high concentration of Saccharomyces cerevisiae on the proliferation of hemocytes in male shrimp Cryphiops caementarius. The shrimp $(5.3 \pm 1.2 \mathrm{~cm}$ of total length and $7.4 \pm 2.9 \mathrm{~g}$ of total weight) were collected from the Pativilca River (Peru). Each shrimp was kept in a container installed inside the aquarium (six shrimp per aquarium). A control diet (3\% yeast) and three experimental diets $(6,9$ and $12 \%$ yeast) were used with two repetitions per treatment during 28 days of culture. The total number of hemocytes was higher $(\mathrm{p}<0.05)$ with $6 \%$ yeast $\left(134.75 \times 10^{5} \mathrm{cells} / \mathrm{ml}\right)$, as well as the number of granulocytes $\left(31.44 \times 10^{5} \mathrm{cell} / \mathrm{s} / \mathrm{ml}\right)$ and semigranulocytes $\left(102.44 \times 10^{5}\right.$ cells $\left./ \mathrm{ml}\right)$. The number of hyalinocytes decreased in all treatments and remained between 0.31 and $1.56 \times 10^{5} \mathrm{cells} / \mathrm{ml}$. The number of atypical hemocytes remained low in all treatments and without differences with the baseline $(0.31$ $\left.\mathrm{x} 10^{5} \mathrm{cells} / \mathrm{ml}\right)$. The diet with $6 \%$ yeast increased $(\mathrm{p}<0.05)$ the total number of hemocytes and the granulocyte and semigranulocyte hemocytes in C. caementarius male shrimps. In contrast, diets with 9 and $12 \%$ of yeast affected $(\mathrm{p}<0.05)$ the proliferation of total and differential hemocytes.

Key words: Saccharomyces, Cryphiops; haemocytes; shrimp; hematopoiesis

\section{INTRODUCCIÓN}

El camarón Cryphiops caementarius (Molina 1782) se distribuye en la vertiente occidental de los Andes, desde el río Taymí en Perú (Méndez, 1981) hasta el río Maipo en Chile (Jara, 1997), pero abunda en los ríos Cañete, Ocoña, Majes-Camaná y Tambo en Perú (Zacarías y Yépez, 2015; Wasiw y Yépez, 2017). La extracción de camarón al 2017 fue de $996 \mathrm{t}$ (Produce, 2018), cuyo principal mercado es la ciudad de Lima, Perú, por la alta demanda en gastronomía. Esta especie de camarón tiene alto valor económico y es prioritaria para cultivo comercial (Brack, 2000); sin embargo, investigaciones sobre parámetros inmunológicos de la especie son escasos.

El sistema inmune de los crustáceos está basado en efectores celulares y humorales, que se conjugan para eliminar microorganismos infecciosos, donde los hemocitos circulantes que constituyen la fracción celular de la hemolinfa realizan fagocitosis, encapsulación, formación de nódulos y producen citotoxicidad (Bachere, 2000; Rendón y Balcázar, 2003; Cerenius et al., 2010; Fonseca et al., 2013). El número total de hemocitos (NTH) y el número diferencial de hemocitos (NDH) de los crustáceos cambian de acuerdo con la especie, sexo, estación de año, enfermedades y condiciones del ambiente, entre otros (Johansson et al., 2000; Gelibolu et al., 2009; Pacheco et al., 2012).

Los crustáceos carecen de una verdadera respuesta inmune adaptativa $y$, por tanto, tienen que depender de mecanismos inmunes innatos muy eficientes en donde los hemocitos desempeñan un papel clave (Lin y Söderhäll, 2011). Los componentes de las paredes de bacterias Gram negativas (lipopolisacáridos), Gram positivas (péptidoglicanos) y de hongos, levaduras y algas ( $\beta$-glucanos), estimulan el sistema inmune de los crustáceos (Rendón y Balcázar, 2003). La formación y desarrollo de hemocitos maduros implica proliferación y diferenciación de células en el tejido hematopoyético (Lin y Söderhäll, 2011). Los inmunoestimulantes e inmunomoduladores se 
incorporan a la dieta para uso continuo porque no generan resistencia ni acostumbramiento y no demandan energía (Berger, 2000); sin embargo, es necesario conocer la dosis adecuada que incremente la respuesta inmune y evite estrés fisiológico en los camarones (Campa-Córdova et al., 2010).

La levadura Saccharomyces cerevisiae no solo aporta $\beta$-glucanos y mananooligosacáridos, sino también con superóxido dismutasa que incrementa la actividad antioxidante y estimula la proliferación de hemocitos circulantes (Pacheco et al., 2012). Subramanian et al. (2012) adicionaron 10\% de $S$. cerevisiae en la dieta de Penaeus monodon y los animales incrementaron sus hemocitos totales, la fenoloxidasa y mostraron alta actividad antibacterial. De esta manera, los camarones con mayor número de hemocitos son resistentes a infecciones bacterianas (Le Moullac et al., 2000). En $C$. caementarius, Cornejo et al. (2015) utilizaron solo $3 \%$ de $S$. cerevisiae en la dieta y lograron incrementar el número total de hemocitos, así como los hemocitos granulocitos y semigranulocitos. Sin embargo, no se conoce la respuesta hemocitaria de este camarón alimentado con otras concentraciones de levadura. Por consiguiente, el objetivo del estudio fue evaluar el efecto de dietas con alta concentración de $S$. cerevisiae sobre la proliferación de hemocitos en camarones $C$. caementarius machos.

\section{Materiales Y Métodos}

\section{Los Organismos}

Los camarones machos se colectaron del río Pativilca, cerca del Centro Poblado Huayto $\left(10^{\circ} 39^{1} 50^{11} \mathrm{~S}, 77^{\circ} 40^{1} 09^{11} \mathrm{~W}\right)$, Lima, Perú. Para el transporte de camarones se empleó el sistema utilizado por Reyes (2016), donde cada camarón se introdujo en un vaso plástico de $200 \mathrm{ml}$ (con agujeros para el flujo de agua), acondicionados en recipientes de plástico (45 l) con agua del mismo río, airea- ción constante y 50 camarones por recipiente. El transporte terrestre duró $4.5 \mathrm{~h}$ y no hubo mortalidad. Los camarones de la especie $C$. caementarius se reconocieron según Méndez (1981) y el sexo de los machos se verificó por la presencia de gonóporos en los coxopoditos del quinto par de periópodos (Reyes et al., 2018). Los camarones se aclimataron durante siete días en el mismo sistema de transporte y se alimentaron con balanceado (30\% de proteína cruda).

\section{Sistema de Cultivo}

El sistema de cultivo fue el empleado por Reyes (2016), que consistió en ocho acuarios de vidrio $(0.60 \mathrm{~m}$ de largo, $0.31 \mathrm{~m}$ de ancho, $0.35 \mathrm{~m}$ de alto) de 551 con sistema de recirculación de agua tipo air-water-lift y filtro biológico percolador $(1.5 \mathrm{l} / \mathrm{min})$. Dentro de cada acuario (unidad experimental) se instalaron seis recipientes de plástico transparente con tapa (19 cm de diámetro, $284 \mathrm{~cm}^{2}$ ) que se dispusieron en dos grupos de tres niveles. Los recipientes tuvieron aberturas en sus paredes para permitir el flujo de agua y un tubo de PVC de $1 / 2$ pulgada de diámetro que sobresalió $10 \mathrm{~cm}$ sobre el nivel del agua, por donde se introdujeron los pellets de alimento. En cada recipiente se sembró un camarón, que equivalió a seis camarones por acuario (32 camarones $/ \mathrm{m}^{2}$ ). Se emplearon 48 camarones machos $(5.3 \pm 1.2 \mathrm{~cm}$ de longitud total y $7.4 \pm 2.9 \mathrm{~g}$ de peso total), con apéndices cefalotorácicos completos y en estados de muda entre $\mathrm{C}$ y $\mathrm{D}_{1}$ (Promwikorn et al., 2004).

\section{Alimento}

La levadura comercial (levadura seca instantánea masa dulce de Fleischmann ${ }^{\circledR}$ ) tuvo como ingredientes a $S$. cerevisiae, monoestearato de sorbitán y ácido ascórbico. Esta levadura se activó con el método de Martínez-Rodríguez et al. (2001) y Perdomo et al. (2004), con modificaciones en la incubación $\left(37^{\circ} \mathrm{C}\right.$ por $\left.24 \mathrm{~h}\right)$. Luego se centrifugó ( $5000 \mathrm{rpm}$ por $15 \mathrm{~min}$ ) y el sedimento húmedo se utilizó como aditivo en las 
dietas. Se empleó la dieta estándar $30 \%$ de proteína cruda, $8.1 \%$ de lípidos y $4.6 \%$ de fibra, con $2600 \mathrm{kcal} / \mathrm{g}$ ) formulada específicamente para C. caementarius (Reyes, 2016). Los pellets de la dieta estándar se molieron en mortero hasta obtener polvo fino; luego, se adicionó el sedimento de levadura activada de acuerdo con cada tratamiento y se roció agua destilada para formar una pasta que se peletizó a baja temperatura en una máquina de procesamiento para carne molida. Las hebras de alimento de $0.3 \mathrm{~mm}$ de diámetro se secaron en estufa $\left(40{ }^{\circ} \mathrm{C}\right.$ por $\left.24 \mathrm{~h}\right) \mathrm{y}$ luego se quebraron hasta obtener pellets de 5 mm de longitud. Se emplearon tres dietas experimentales $(6,9$ y $12 \%$ de levadura activada) y una dieta control ( $3 \%$ de levadura activada, según Cornejo et al., 2015), cada una con dos repeticiones. La ración diaria (08:00 y 19:00) fue del $6 \%$ del peso húmedo por camarón, que se distribuyó en iguales proporciones durante seis días a la semana.

\section{Hemolinfa}

La extracción de hemolinfa se realizó de dos camarones por tratamiento y por muestreo al inicio del experimento y luego cada siete días durante cuatro semanas de cultivo. A cada camarón se le extrajo hemolinfa una sola vez. Los camarones seleccionados se les mantuvo en ayunas por 12 $\mathrm{h}$ antes de la extracción de hemolinfa. Para la extracción, cada camarón se mantuvo por 5 min en un recipiente plástico (4 1) con agua dulce preaireada; se retiró al camarón, se le envolvió con tela y se descubrió la zona dorsal entre el cefalotórax y el abdomen, y con una jeringa de $1 \mathrm{ml}$ (insulin syringe $26 \mathrm{G} 1 / 2$ ) se extrajo la hemolinfa $(\sim 0.10 \mathrm{ml})$ del seno pericárdico.

Para determinar el NTH y el NDH se utilizó $20 \mu 1$ de hemolinfa y se adicionó a un microtubo (Eppendorf) con $80 \mu 1$ de solución Alsever (van de Braak, 2002). El conteo de hemocitos se realizó en cámara de Neubauer bajo microscopio LEICA DM LS2 de luz convencional de campo luminoso provisto de contraste de fases.
El NTH y el NDH (hemocitos granulocitos [HG], hemocitos semigranulocitos [HSG], hemocitos hialinos [HH] y hemocitos atípicos [HA]) se determinaron según Hose et al. (1990) con las fórmulas de Jussila (1997): $\mathrm{NTH}($ células $/ \mathrm{ml})=\left(\mathrm{Hc}^{*} \mathrm{D} * \mathrm{C}\right) / 0.4 \mathrm{y}$ $\mathrm{NDH}($ células $/ \mathrm{ml})=\left(\mathrm{Hd}^{*} \mathrm{D}^{*} \mathrm{C}\right) / 0.4$, donde $\mathrm{Hc}$ es el número total de hemocitos contados, $\mathrm{D}$ el factor de dilución de la hemolinfa, $\mathrm{C}$ la conversión del factor $\mathrm{x} 0.1 \mathrm{~mm}^{3} \mathrm{x} \mathrm{ml}(1000) \mathrm{y}$ Hd el número de hemocitos diferenciales contados.

\section{Calidad del Agua}

Los desechos sólidos acumulados en los acuarios se extrajeron todos los días con sifón. El 30\% del agua de cada acuario se renovó dos veces por semana. El agua de los acuarios se monitoreó cada semana y se determinó oxígeno disuelto y temperatura con Oxímetro Hatch LDO $( \pm 0.01 \mathrm{mg} / 1 ; \pm$ $\left.0.01{ }^{\circ} \mathrm{C}\right), \mathrm{pH}$ con $\mathrm{pH}$-metro digital $110( \pm 0.01$ unidades) y amonio total y nitritos con test colorimétrico Nutrafin $( \pm 0.5 \mathrm{mg} / \mathrm{l})$.

\section{Análisis Estadístico}

Los resultados se sometieron a la prueba de Kolmogorov-Smirnov para determinar la normalidad de datos. Se aplicó el análisis de varianza de una vía y la prueba de Duncan, así como la prueba t Student para comparar el NTH y NDH basales con los obtenidos en cada muestreo. En todos los casos se empleó el nivel de significancia del 5\%. Los datos se expresaron como media \pm desviación estándar. Todos los análisis se efectuaron con el software SPSS v. 23 para Windows.

\section{Resultados}

\section{Calidad del Agua}

Los parámetros de calidad del agua fueron similares entre tratamientos. La temperatura varió entre 25.5 y $26.8^{\circ} \mathrm{C}$, el pH entre 7.05 y 7.21 , el oxígeno disuelto entre 3.70 y 
$5.40 \mathrm{mg} / \mathrm{l}$, los nitritos fueron de $0.10 \mathrm{mg} / \mathrm{l} \mathrm{y}$ no se detectó amonio total.

\section{Número Total de Hemocitos}

E1 NTH basal de los camarones fue de $11.06 \pm 1.50 \times 10^{5} \mathrm{cé} 1 / \mathrm{ml}$, habiendo un incremento abrupto en los primeros siete días $(\mathrm{p}<0.05)$ en todos los tratamientos. El NTH de los camarones alimentados con $6 \%$ de levadura incrementó $(\mathrm{p}<0.05)$ a los 21 días $\left(88.31 \pm 5.26 \times 10^{5}\right.$ cél $\left./ \mathrm{ml}\right)$ y 28 días de cultivo $\left(134.75 \pm 2.30 \times 10^{5}\right.$ cél $\left./ \mathrm{ml}\right)$. El NTH de aquellos alimentados con $9 \%$ de levadura incrementó $(\mathrm{p}<0.05)$ en los primeros siete días, pero disminuyó en el tiempo. En los demás tratamientos, el NTH varió con el tiempo y no superaron los $78 \times 10^{5}$ cél/ml (Figura 1a).

\section{Hemocitos Granulocitos}

El número de HG basal de los camarones fue de $3.19 \pm 0.62 \times 10^{5}$ cél $/ \mathrm{ml}$ y hubo un incremento abrupto $(\mathrm{p}<0.05)$ en los primeros siete días en todos los tratamientos. Los camarones alimentados con $6 \%$ de levadura incrementaron $(\mathrm{p}<0.05)$ los $\mathrm{HG}$ a los 14 días $\left(16.06 \pm 1.68 \times 10^{5} \mathrm{cé} 1 / \mathrm{ml}\right), 21$ días $(18.06 \pm$ $0.80 \times 10^{5}$ cél $\left./ \mathrm{ml}\right)$ y 28 días de cultivo $(31.44$ $\left.\pm 3.45 \times 10^{5} \mathrm{cé} 1 / \mathrm{ml}\right)$. Los HG fluctuaron en el tiempo en los otros tratamientos y no superaron los $23 \times 10^{5} \mathrm{cé} / \mathrm{ml}$ (Figura 1b).

\section{Hemocitos Semigranulocitos}

El número de HSG basal de los camarones fue de $6.00 \pm 1.24 \times 10^{5} \mathrm{cé} 1 / \mathrm{ml}$, y hubo un incremento abrupto a los siete días $(\mathrm{p}<0.05)$ en todos los tratamientos. Los camarones alimentados con $6 \%$ de levadura incrementaron $(\mathrm{p}<0.05)$ los HSG a los 21 días $\left(69.13 \pm 6.898 \times 10^{5}\right.$ cél $\left./ \mathrm{ml}\right)$ y 28 días de cultivo $\left(102.44 \pm 5.57 \times 10^{5}\right.$ cél $\left./ \mathrm{ml}\right)$. Los HSG fluctuaron en el tiempo en los demás tratamientos y no superaron los $57 \times 10^{5}$ cél $/ \mathrm{ml}$ (Figura 2a).

\section{Hemocitos Hialinocitos}

El número de HH basal de los camarones fue de $1.56 \pm 0.44 \times 10^{5}$ cél $/ \mathrm{ml}$. Los HH de aquellos alimentados con $6 \%$ de levadura incrementaron $(\mathrm{p}<0.05)$ a los siete días de cultivo $\left(3.19 \pm 0.62 \times 10^{5}\right.$ cél $\left./ \mathrm{ml}\right)$, para luego disminuir con el tiempo hasta $0.39 \pm 0.09 \mathrm{x}$ $10^{5}$ cél $/ \mathrm{ml}$. A los 14 días se registró incremento abrupto $(\mathrm{p}<0.05)$ de los $\mathrm{HH}$ en los camarones alimentados con $3 \%(6.38 \pm 0.53$
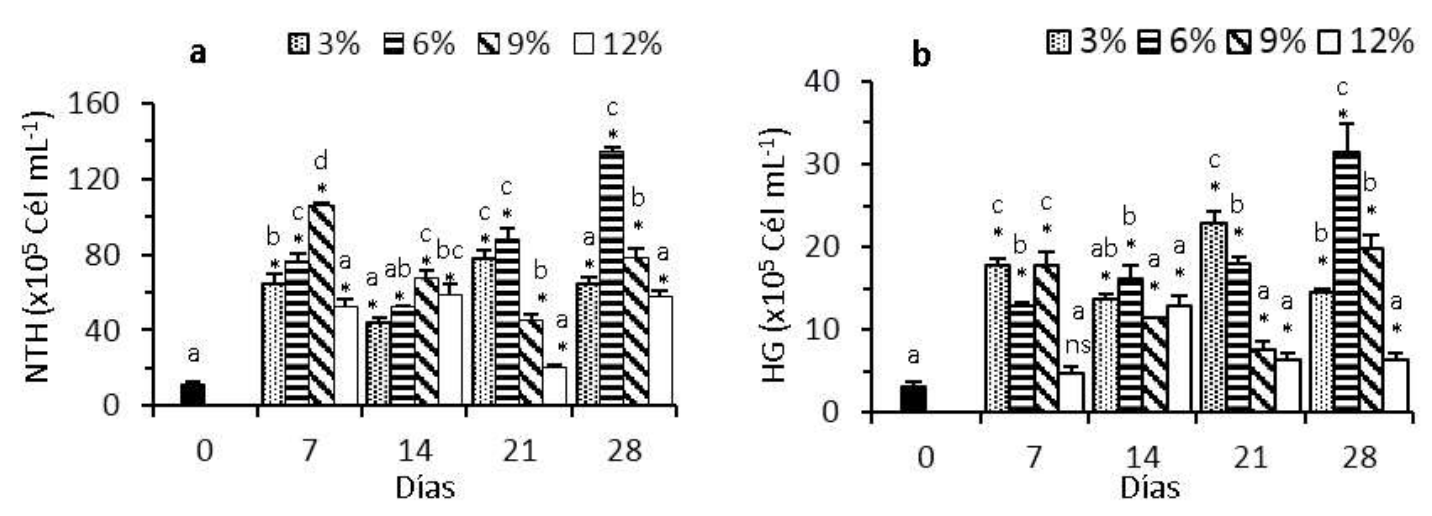

Figura 1. Machos C. caementarius alimentados con altas concentraciones de levadura $S$. cerevisiae en la dieta. a) Número total de hemocitos (NTH). b) Número de hemocitos granulocitos (HG). Letras diferentes sobre las barras, por cada muestreo, indican diferencias significativas $(\mathrm{p}<0.05)$. El asterisco indica diferencia significativa $(\mathrm{p}<0.05)$ con relación al valor inicial; ns es no significativo al valor inicial 

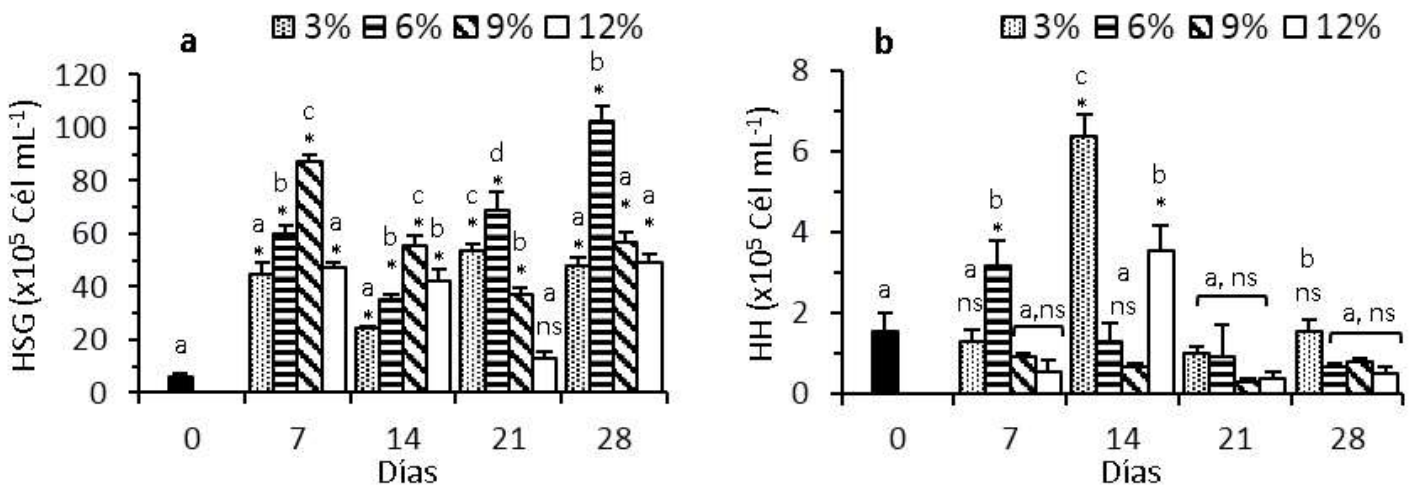

Figura 2. Machos C. caementarius alimentados con altas concentraciones de levadura $S$. cerevisiae en la dieta. a) Número de hemocitos semigranulocitos (HSG). b) Número de hemocitos hialinos (HH). Letras diferentes sobre las barras, por cada muestreo, indican diferencias significativas $(\mathrm{p}<0.05)$. El asterisco indica diferencia significativa $(p<0.05)$ con relación al valor inicial; $n$ s es no significativo al valor inicial

x $10^{5}$ cél $\left./ \mathrm{ml}\right)$ y $12 \%\left(3.56 \pm 0.62 \times 10^{5}\right.$ cél $/$ $\mathrm{ml}$ ) de levadura, para luego disminuir de la misma manera a través del tiempo. Los $\mathrm{HH}$ de los alimentados con $9 \%$ de levadura se mantuvieron bajos $\left(\sim 0.8 \times 10^{5} \mathrm{cél} / \mathrm{ml}\right)$ durante el periodo experimental (Figura $2 b$ ).

\section{Hemocitos Atípicos}

El número de HA basal de los camarones fue de $0.31 \pm 0.09 \times 10^{5}$ cél $/ \mathrm{ml}$. Los HA se mantuvieron bajos durante los 28 días de cultivo y sin diferencias con los HA basal; excepto en aquellos alimentados con $12 \%$ de levadura cuyos HA a los 21 días fue alto $(0.56$ $\left.\pm 0.09 \times 10^{5} \mathrm{cé} / \mathrm{ml}\right)$ y mucho más alto a los 28 días $\left(1.13 \pm 0.18 \times 10^{5}\right.$ cél $\left./ \mathrm{ml}\right)$ con relación al basal y con los demás tratamientos que fluctuaron entre $0.19 \pm 0.09 \times 10^{5}$ cé $1 / \mathrm{ml}$ y $0.38 \pm 0.18 \times 10^{5}$ cél $/ \mathrm{ml}$ (Figura 3 ).

\section{Número Relativo de Hemocitos}

La alimentación de camarones durante 28 días con diferentes proporciones de levadura en la dieta ocasionó incremento relativo $(\mathrm{p}<0.05)$ en el número de los HSG $(73-86 \%)$ y disminución $(\mathrm{p}<0.05)$ de los HG (de $23 \mathrm{a}$ $11 \%$ ) con relación a los hemocitos del basal

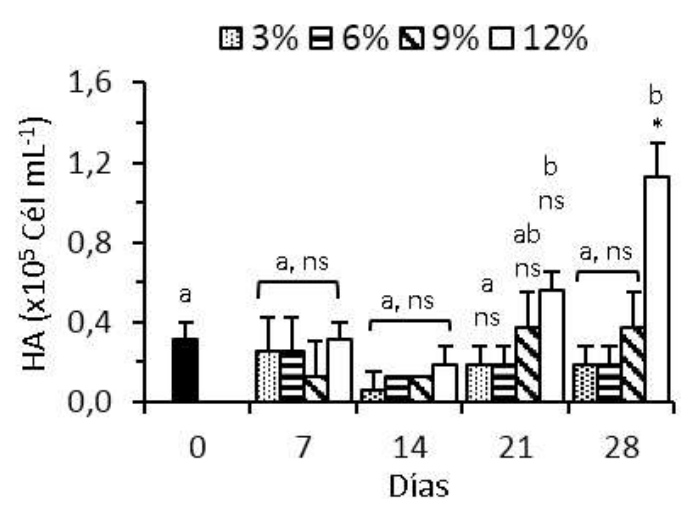

Figura 3. Número de hemocitos atípicos (HA) en machos de $C$. caementarius alimentados con altas concentraciones de levadura $S$. cerevisiae en la dieta. Letras diferentes sobre las barras, por cada muestreo, indican diferencias significativas $(\mathrm{p}<0.05)$. El asterisco indica diferencia significativa con relación al valor inicial $(p<0.05)$; ns es no significativo al valor inicial

(54 y $28 \%$, respectivamente). En cambio, se obtuvo diminución $(\mathrm{p}<0.05)$ de los $\mathrm{HH}(\mathrm{de}$ 2.43 a $0.51 \%$ ) y HA (de 0.14 a $1.96 \%$ ) con relación a los hemocitos del basal (14.11 y $2.80 \%$, respectivamente) (Cuadro 1). 
Cuadro 1. Número relativo (\%) de hemocitos de machos C. caementarius alimentados durante 28 días con altas concentraciones de levadura Saccharomyces cerevisiae en la dieta

\begin{tabular}{lccccc}
\hline \multirow{2}{*}{ Hemocitos } & Hemocitos & \multicolumn{4}{c}{ Levadura } \\
\cline { 3 - 6 } & basales & $3 \%$ & $6 \%$ & $9 \%$ & $12 \%$ \\
\hline Granulocitos (HG) & $28.32 \pm 2.23^{\mathrm{a}}$ & $22.70 \pm 0.53^{\mathrm{ab}}$ & $23.36 \pm 2.96^{\mathrm{ab}}$ & $24.80 \pm 1.37^{\mathrm{ab}}$ & $11.09 \pm 0.50^{\mathrm{c}}$ \\
Semigranulocitos (HSG) & $54.22 \pm 3.47^{\mathrm{a}}$ & $74.57 \pm 0.95^{\mathrm{b}}$ & $76.00 \pm 2.84^{\mathrm{b}}$ & $73.01 \pm 0.21^{\mathrm{b}}$ & $86.10 \pm 0.93^{\mathrm{c}}$ \\
Hialinocitos (HH) & $14.63 \pm 6.13^{\mathrm{a}}$ & $2.45 \pm 0.54^{\mathrm{b}}$ & $0.51 \pm 0.05^{\mathrm{b}}$ & $1.04 \pm 0.05^{\mathrm{b}}$ & $0.87 \pm 0.25^{\mathrm{b}}$ \\
Atípicos (HA) & $2.83 \pm 0.43^{\mathrm{a}}$ & $0.29 \pm 0.12^{\mathrm{c}}$ & $0.14 \pm 0.06^{\mathrm{c}}$ & $0.49 \pm 0.26^{\mathrm{c}}$ & $1.95 \pm 0.18^{\mathrm{b}}$ \\
\hline
\end{tabular}

$a, b, c$ Superíndices diferentes dentro de filas indican diferencias significativas $(p<0.05)$

\section{Discusión}

La calidad del agua de cultivo de $C$. caementarius fue similar entre tratamientos y estuvieron dentro de lo registrado para el hábitat de la especie (Wasiw y Yépez, 2017). Además, fue semejante a las condiciones normales de laboratorio donde se experimenta con la especie en el mismo sistema de cultivo (Cornejo et al., 2015; Reyes, 2016). En consecuencia, la variación de los hemocitos de los camarones se debió a la presencia de la levadura $S$. cerevisiae en las dietas.

El NTH basal $\left(11.06 \times 10^{5} \mathrm{cé} 1 / \mathrm{ml}\right)$ y el NDH basal de los HG $\left(3.19 \times 10^{5}\right.$ cél $\left./ \mathrm{ml}\right)$, HSG $\left(6.00 \times 10^{5}\right.$ cél $\left./ \mathrm{ml}\right)$ y HH $\left(1.56 \times 10^{5}\right.$ cél $/ \mathrm{ml})$ de $C$. caementarius fueron aproximadamente tres veces mayor que los obtenidos previamente (Cornejo et al., 2015), lo que sugiere que los camarones del presente estudio estuvieron en mejores condiciones inmunológicas o que procedieron de un ambiente estable. Sin embargo, no se determinó la calidad del agua del río de procedencia de los camarones en ambas investigaciones. Las variaciones en los hemocitos circulantes en Callinectes sapidus se deben a la temporada y a las condiciones ambientales, entre otros factores (Gelibolu et al., 2009).
Las concentraciones de levadura en la dieta ocasionaron incrementos abruptos del NTH, HG y HSG de C. caementarius en los primeros siete días de alimentación, lo que significa que los camarones respondieron $\mathrm{r}$ [apidamente a la levadura proporcionada. Sin embargo, en estos primeros días, los mayores valores en el NTH y HSG se obtuvieron con $9 \%$ de levadura $(\mathrm{p}<0.05)$, lo que sugiere que esta sería la concentración de levadura en la dieta para estimular de manera rápida la proliferación de hemocitos de la especie, aunque este valor disminuyó a los 14 días, probablemente debido al exceso de levadura que afectó la hematopoyesis, como ha sido sugerido por Chang et al. (2000) en P. monodon.

En cambio, la dieta con $6 \%$ de levadura ocasionó incremento $(\mathrm{p}<0.05)$ del NTH desde los 21 hasta los 28 días de cultivo (134.75 x $10^{5}$ cél $/ \mathrm{ml}$ ), tiempo en que se alcanzaría la máxima cantidad de hemocitos totales registrados para C. caementarius, lo que indicaría un mejor estado inmunológico respecto a los valores basales, y a lo informado por Cornejo et al. (2015) aunque con 3\% de levadura en la dieta $\left(33.05 \times 10^{5}\right.$ cél $\left./ \mathrm{ml}\right)$. El incremento de hemocitos totales se relaciona con el estado nutricional de los crustáceos, cuya respuesta inmune se refuerza con los inmunoestimulantes de la levadura (Le Moullac et al., 2000) y que en Litopenaeus 
vannamei les permite resistir infecciones bacterianas y virales (Campa y Luna, 2011). Además, las levaduras son activadores, moduladores y fortificadores del sistema inmune en diversos organismos por la presencia de $\beta$-glucanos, mananooligosacáridos y otros componentes de la pared celular (ZhenMing et al., 2010) que estimulan la proliferación de hemocitos como en $L$. vannamei (Campa-Córdova et al., 2010).

Las variaciones en el NTH de los crustáceos dependen del sexo, temporada, condiciones ambientales, enfermedades (Johansson et al., 2000; Gelibolu et al., 2009; Pacheco et al., 2012) y de la respuesta inmune de cada especie de crustáceo (Song y Hsieh, 1994; Matozzo y Marin, 2010). En $C$. caementarius, el NTH máximo fue de 134.75 x $10^{5}$ cél $/ \mathrm{ml}$ estimulado con $6 \%$ de levadura en la dieta. Alto NTH se registra en Eriocheir sinensis en machos y en hembras (Lv et al., 2014) y en P. monodon (van de Braak, 2002). En cambio, bajo NTH se informa en Panulirus argus (Li y Shields, 2007), Carcinus aestuarii (Matozzo y Marin, 2010) у C. sapidus (Gelibolu et al., 2009).

Las diferentes proporciones de levadura en la dieta ocasionaron en $C$. caementarius incremento relativo de los HSG (de 73 a 86\%) en relación con el basal (54\%); en cambio, disminuyeron los HG (de 25 a $11 \%)$ y $\mathrm{HH}$ (de 2 a $0.5 \%$ ) con relación al valor basal ( 28 y $14 \%$, respectivamente). Esto demuestra que la levadura estimula principalmente la proliferación de HSG, lo cual es necesario para el camarón, toda vez que este tipo de hemocitos participa en fagocitosis, como lo sucedido en $M$. rosenbergii y $M$. acanthurus (Gargioni y Barracco, 1998). En P. leniusculus, los HSG constituyen el $65 \%$ de las células (Lin y Söderhäll, 2011). En $P$. argus, los HSG representan el 58\%, los HG el 27\% y los HH el 15\% (Li y Shields, 2007), similar a Procambarus clarkii (Din et al., 2012).
La dieta con 6\% de levadura fue la única que estimuló la proliferación de HG (31.44 x $10^{5}$ cél $\left./ \mathrm{ml}\right)$ y HSG $\left(102.44 \times 10^{5} \mathrm{cél} / \mathrm{ml}\right) \mathrm{n}$ C. caementarius durante 28 días de cultivo, en relación con $3 \%$ de levadura $\left(23 \times 10^{5}\right.$ cél $/ \mathrm{ml}), 9$ y $12 \%$ de levadura ( 57 x $10^{5}$ cél/ $\mathrm{ml})(\mathrm{p}<0.05)$. Estos resultados indican que $6 \%$ de levadura sería la máxima concentración para emplear en la dieta de C. caementarius para estimular la proliferación de los HGy HSG.

Los $\mathrm{HH}$ en $C$. caementarius de todos los tratamientos fluctuaron en el tiempo con respecto al basal $\left(1.56 \times 10^{5}\right.$ cél $\left./ \mathrm{ml}\right)$, pero disminuyeron hasta $\sim 0.40 \times 10^{5} \mathrm{cé} 1 / \mathrm{ml}$, aunque estos valores fueron más altos que lo informado por Cornejo et al. (2015) de $\sim 0.11 \mathrm{x}$ $10^{5}$ cél $/ \mathrm{ml}$. Esta variación de los HH durante el tiempo de cultivo podría ser ocasionado por alguna enfermedad orgánica o alguna infección que no fue perceptible a simple vista y que explicaría el incremento abrupto de los HH que se observó a los siete días con $6 \%$ de levadura y a los 14 días con 3 y $12 \%$ de levadura en la dieta. Esto se sustenta por el hecho de que los $\mathrm{HH}$ de los crustáceos decápodos tienen capacidad fagocítica y participan en la coagulación (Raa, 2000; Johansson et al., 2000; Montesdeoca et al., 2002; Lv et al., 2014).

La variación de los HA en C. caementarius alimentado hasta con $9 \%$ de levadura fue similar con el basal $\left(0.31 \times 10^{5}\right.$ cél $\left./ \mathrm{ml}\right)$ y a lo reportado $\left(0.28 \times 10^{5}\right.$ cél $\left./ \mathrm{ml}\right)$ por Cornejo et al. (2015). En cambio, el incremento abrupto de los HA observado a los 21 y 28 días de cultivo solo en aquellos alimentados con $12 \%$ de levadura indicaría alteraciones en la hematopoyesis por exceso de levadura que debe haber afectado el estado fisiológico e inmunológico de los camarones. En $L$. vannamei, la alta concentración de HA se relaciona con deficiente estado inmunitario, baja capacidad de reemplazo por hemocitos nuevos y libre circulación de células inmunitarias desgastadas o apoptóticas (Maldonado, 2003). 
La disminución del NTH, HG y HSG en camarones machos $C$. caementarius alimentados con 9 y $12 \%$ de levadura desde los 21 días de cultivo es evidencia de que ambas concentraciones de levadura fueron elevadas que afectaron la proliferación de hemocitos y ocasionaron el incremento de los HA. Según Chang et al. (2000), una sobredosis de levadura en $P$. monodon causa fatiga inmune. En cambio, el $6 \%$ de levadura en la dieta ocasionó en $C$. caementarius mayor incremento del NTH, HSG y disminución de HA con relación a lo informado por Cornejo et al. (2015) con 3\% de levadura. Sin embargo, es conveniente complementar dichos resultados con otros parámetros inmunológicos y, además, realizar el desafío con bacterias patógenas.

\section{Conclusiones}

- La dieta con $6 \%$ de levadura incrementó $(\mathrm{p}<0.05)$ el número total de hemocitos y los hemocitos granulocitos y semigranulocitos de camarones machos de $C$. caementarius, durante 28 días de cultivo.

- Las dietas con 9 y $12 \%$ de levadura afectaron $(\mathrm{p}<0.05)$ la proliferación de hemocitos totales y diferenciales en los camarones machos de C. caementarius.

\section{Literatura Citada}

1. Bachere E. 2000. Shrimp immunity and disease control. Aquaculture 191: 3-11. doi: 10.1016/S0044-8486(00)00413-0

2. Berger C. 2000. Aportes de la bio-tecnología a la alimentación y a la inmunoestimulación de camarones peneidos. En: V Simposio Internacional de Nutrición Acuícola. Mérida, Yucatán.

3. Brack A. 2000. Perú biodiversidad y comercio. Situación actual y potencial. Comité Biocomercio Perú. [Internet]. Disponible en: http://repositorio.promperu.gob.pe/repositorio/bitstream/ handle/123456789/908/Per\%- c3\%ba_biodiversidad_biocomercio_situaci\%c3\% b3n_actual_potencial_2000_keyword_principal.pdf?-sequen-ce $=3$ \&is-Allowed $=\mathrm{y}$

4. Campa A, Luna A. 2011. Respuesta inmune en camarón blanco, Litopenaeus vannamei, expuesto a infecciones bacterianas y virales. En: XI Simposio Internacional de Nutrición Acuícola. México.

5. Campa-Córdova AI, HernándezSalmerón A, Ascencio-Valle $F$, Aguirre-Guzman G. 2010. Respuesta inmune y antioxidante en camarón blanco Litopenaeus vannamei, expuesto a inmunoestimulantes y probióticos. En: $\mathrm{X}$ Simposio Internacional de Nutrición Acuícola. México.

6. Cerenius L, Jiravanichpaisal P, Liu H, Söderhäll I. 2010. Crustacean immunity. Adv Exp Med Biol 708: 239259. doi: 10.1007/978-1-4419-8059-5_13

7. Chang CF, Chen HY, Su MS, Liao IC. 2000. Immunomodulation by dietary $\beta$ 1,3-glucan in the brooders of the black tiger shrimp Penaeus monodon. Fish Shellfish Immun 10: 505-514. doi: 10.1006/fsim.2000.0266

8. Cornejo J, Pérez L, Reyes W. 2015. Effect of Saccharomyces cerevisiae yeast in the diet of male shrimp Cryphiops caementarius (Crustacea, Palaemonidae) on total and differential hemocytes count. Rev Bio Ciencias 3:173-186. 10.15741/revbio.03.03.04

9. Din Z, Du J, Ou J, Li W, Wu T, Xiu Y, Meng Q, et al. 2012. Classification of circulating hemocytes from the red swamp crayfish Procambarus clarkii and their susceptibility to the novel pathogen Spiroplasma eriocheiris in vitro. Aquaculture 356-357: 371-380. doi: 10.1016/j.aquaculture.2012.04.042

10. Fonseca E, González R, Rico R. 2013. Sistema inmune de los camarones. AquaTIC 38: 68-84.

11. Gargioni R, Barracco MA. 1998. Hemocytes of the palaemonids Macrobrachium rosenbergii and $M$. acanthurus, and of the penaeid Penaeus 
paulensis. J Morphol 236: 209-221. doi: 10.1002 / ( S IC I ) 1097 - 4687 (199806)236:3<209::AID-JMOR4>3.0.CO;2-Y

12. Gelibolu S, Türeli C, Sahan A. 2009. Determination of haemocytes amount and haemocytes type in mature blue crab (Callinectes sapidus, Rathbun, 1896) captured in Akyatan lagoon (Karatas/AdanaTurkey). J Fisheries Sci 3: 181-186.

13. Hose JE, Martin GG, Gerard AS. 1990. A decapod hemocyte classification scheme integrating morphology, cytochemistry and function. Biol Bull 178: 33-45.

14. Jara CG. 1997. Antecedentes sobre el desarrollo de la carcinología en Chile. Investigaciones Marinas 25: 245-254.

15. Johansson M, Keyser P, Sritunyalucksana K, Söderhäll I. 2000. Crustacean haemocytes and haematopoiesis. Aquaculture 191: 45-52. doi: 10.1016/S0044-8486(00)00418-X

16. Jussila J. 1997. Physiological responses of astacid and parastacid crayfishes (Crustacea: Decapoda) to conditions of intensive culture. PhD Thesis. Western Australia: University of Kuopio. 138 p.

17. Le Moullac G, Phillip L, Saulnier D, Goarant C, Dehasque M. 2000. Principios y problemas envueltos en la evaluación de immunoestimulantes en juveniles de camarón. En: IV Simposio Internacional de Nutrición Acuícola. México.

18. Li C, Shields JD. 2007. Primary culture of hemocytes from the Caribbean spiny lobster, Panulirus argus, and their susceptibility to Panulirus argus Virus 1 (PaV1). J Invertebr Pathol 94: 48-55. doi: 10.1016/j.jip.2006.08.011

19. Lin X, Söderhäll I. 2011. Crustacean hematopoiesis and the astakine cytokines. Blood 117: 6417-6424. doi: 10.1182/blood-2010-11-320614

20. LvS, Xu J, Zhao J, Yin N, Lu B, Li S, Chen Y, Xu H. 2014. Classification and phagocytosis of circulating haemocytes in Chinese mitten crab (Eriocheir sinensis) and the effect of extrinsic stimulation on circulating haemocytes in vivo. Fish Shellfish Immun 39: 415-422.
21. Maldonado M. 2003. Respuesta inmunitaria en familias de Litopenaeus vannamei, bajo condiciones de infección con WSSV y el efecto de la adición de $\beta-1,3$ glucanos. Tesis de Magíster. Guayaquil, Ecuador: Instituto Nacional Politécnica del Litoral. $106 \mathrm{p}$.

22. Martínez-Rodriguez AJ, Polo MC, Carrascosa AV. 2001. Structural and ultrastructural changes in yeast cells during autolysis in model wine system ad in sparkling wines. Int J Food Microbiol 71: 45-51. doi: 10.1016/S01681605(01)00554-2

23. Matozzo V, Marin MG 2010. The role of haemocytes from the crab Carcinus aestuarii (Crustacea, Decapoda) in immune responses: a first survey. Fish Shellfish Immun 28: 534-541. doi: 10.1016/j.fsi.2009.12.003

24. Méndez M. 1981. Claves de identificación y distribución de los langostinos y camarones (Crustacea: Decapoda) del mar y ríos de la costa del Perú. Bol Instituto del Mar Perú 5: 1-170.

25. Montesdeoca M, Amano $Y$, Echevarria F, Betancout I, Sotomayor M, Rodríguez J. 2002. La respuesta inmunitaria celular del camarón Litopenaeus vannamei al WSSV y su utilidad en el control de la enfermedad en los estanques. Mundo Acuícola 8: 38-42.

26. Pacheco M, Campa A, Aguirre G, Guzman M, Ascencio F. 2012. Efecto de Debaryomyces hansenii en la respuesta antioxidante de juveniles de camarón blanco Litopenaeus vannamei. Rev MVZ Córdoba 17: 2820-2826.

27. Perdomo MC, Vargas RA, Campos J. 2004. Valor nutritivo de la levadura de cervecería (Saccharomyces cerevisiae) y de sus derivados, extracto y pared celular, en la alimentación aviar. Arch Latinoam Prod Anim 12 :89-95.

28. [Produce]. Ministerio de la Producción. 2018. Anuario estadístico pesquero y acuícola 2016. La actividad productiva del sector en números. Perú. [Internet]. Disponible en: http:// ogeiee.produce.gob.pe/images/Anuario/ Pesca_2017.pdf 
29. Promwikorn W, Kirirat P, Thaweethamsewee P. 2004. Index of molt staging in the black tiger shrimp (Penaeus monodon). Songklanakarin J Sci Technol 26: 765-772.

30. Raa J. 2000. The use of immune stimulants in fish and shellfish feeds. En: V Simposio Internacional de Nutrición Acuícola. Mérida, Yucatán, México.

31. Rendón L, Balcázar J. 2003. Inmunología de camarones: conceptos básicos y recientes avances. AquaTIC 19:27-33.

32. Reyes W, Ferrer K, Sernaqué J. 2018. Dimorfismo sexual del camarón Cryphiops caementarius (Crustacea, Palaemonidae). En: Mem XVIII Congreso Nacional de Estudiantes de Biología. Ancash, Perú.

33. Reyes W. 2016. Effect of culture container on the survival and growth of male Cryphiops caementarius in individualized systems. Rev Bio Ciencias 3: 311325. http://dx.doi.org/10.15741/revbio.03.04 .06

34. Song YL, Hsieh YT. 1994. Immunostimulation of tiger shrimp (Penaeus monodon) hemocytes for generation of microbicidal substances: analysis of reactive oxygen species. Dev Comp Immunol 18: 201-209. doi: 10.1016/0145305X(94)90012-4

35. Subramanian M, Alikunhi NM, Kandasamy K. 2012. Immunostimulatory effect of mangrove-derived marine yeasts in Penaeus monodon. Aquac Res 45: 3889-3396. doi: 10.1111/ j.1365-2109.2012.03235.x

36. van de Braak K. 2002. Haemocitic defence in black tigre shrimp (Penaeus monodon). PhD Thesis. The Netherlands: Wagenningen Institute of Animal Sciences. $159 \mathrm{p}$.

37. Wasiw J, Yépez V. 2017. Evolución de la condición poblacional del camarón Cryphiops caementarius en el río Cañete (2000-2015). Rev Inv Vet Perú 28: 13-32. doi: 10.15381/rivep.v28i1.12942

38. Zacarías S, Yépez V. 2015. Camarón de río Cryphiops caementarius (Molina, 1782) en la costa centro-sur del Perú, 2007. Instituto del Mar del Perú 42: 398-415.

39. Zhen-Ming C, Liu G, Zhao S, Li J, Peng $Y$. 2010. Marine yeasts as biocontrol agents and producers of bioproducts. Appl Microbiol Biot 86: 12271241. doi: 10.1007/s00253-010-2483-9 